\title{
Estudio de un brote de varicela en un pueblo del Uruguay
}

\author{
Jorge Quian R., Alejandra Protasio P., Patricia Dall'Orso V., Mariana Mas G., \\ Claudia Romero O., Nelson Ferreira J. e Isabel Coria R.
}

\section{Varicella outbreak in a village in Uruguay}

A varicella outbreak occurred in a Uruguayan village that introduced the varicella vaccine in 1999 with currently high vaccination rates. Aim: To investigate the cause of the outbreak, vaccine effectiveness, and to describe the measures adopted. Material and Methods: Cases that occurred in the kindergarten and schools in the village were investigated. Vaccination cards were examined, history of chickenpox and clinical characteristic of the current episode were obtained and the outcome of the measures were evaluated. An estimate was made of the vaccine's effectiveness. Results: A total of 37 cases of varicella were reported, 14 occurring in previously vaccinated children, in a 1 total population of 313 children. The global effectiveness of the vaccine was $80 \%$, and $100 \%$ for severe cases. A shift of cases towards older ages was demonstrated; vaccinated children had a trend of less fever and lower number of lesions. Immunization of healthy unvaccinated children, mainly adolescents stopped the outbreak. Comments: The vaccine proved to be highly effectivene. In an outbreak situation immunization policies should consider "catch up" vaccination in non-immunized adolescents without a previous history of varicella.

Key words: Varicella, vaccine, effectiveness, outbreak.

Palabras clave: Varicela, vacuna, efectividad, brote epidémico.

\section{Introducción}

En países con altos índices de vacunación como Uruguay, que alcanza coberturas de más del $90 \%$ a lo largo de los años, la presencia de un brote de una enfermedad prevenible debe ser investigado. Las causas pueden ser varias, como inadecuado lote de vacunas, pérdida de la cadena de frío, coberturas vacunales no tan altas u otras ${ }^{1}$.

Uruguay se ha caracterizado por políticas avanzadas en cuanto a la incorporación de vacunas en el Programa Ampliado de Inmunizaciones (PAI). En 1994 fue el primer país de América Latina en incorporar la vacuna de Haemophilus influenzae tipo b. En 1999 introdujo la vacuna anti-varicela en el PAI, siendo de los primeros países en el mundo en tomar tal decisión. Estados Unidos de América (E.U.A.) lo había hecho en $1995^{2}$.

E1 PAI en Uruguay tiene dos características de importancia en el análisis de los resultados: las vacunas en él incluidas son obligatorias y gratuitas para toda la población; se exige documentar el cumplimiento del PAI para las actividades curriculares (jardín de infantes, escuelas, liceos). Por tanto, se alcanzan elevadas coberturas de vacunación, lo que es distinto a lo sucedido en otros países ${ }^{3}$.

La experiencia con la vacuna anti-varicela en Uruguay ha sido exitosa, aplicada en una sola dosis a los 12 meses de edad, junto al resto de las inmunizaciones correspondientes (DPT/Hib/HB); Tresvírica; anti-polio oral) y se han publicado los resultados de la misma ${ }^{4,5}$, lo que a su vez certifica los altos niveles de cobertura. A partir de marzo de 2008, hubo una modificación; vacuna anti-varicela se sigue administrando a los 12 meses, pero junto a triple viral, anti-polio y neumocóccica heptavalente.

Si bien han aparecido en la literatura médica opiniones de que sería necesaria una segunda dosis de la vacuna, y ya ha sido incorporada en el esquema de E.U.A. ${ }^{6,7}$, Uruguay mantiene el esquema de dosis única.

En julio de 2008, se comunicó la existencia de un brote de varicela en dos pueblos del interior del país: Villa Tambores y Piedra Sola. Dado el interés del tema, ya que la aparición de brotes en países con alto nivel de vacunación debe hacer plantear hipótesis como las mencionadas, los autores decidieron investigar las causas del mismo, en uno de los pueblos, Villa Tambores. La investigación solamente se realizó en niños escolarizados, aunque las denuncias involucraron también adultos y lactantes.

Los objetivos del estudio fueron: describir las características del brote; determinar la efectividad clínica de la vacuna y, por último, analizar las medidas tomadas por la autoridad sanitaria del país, el Ministerio de Salud Pública (MSP).

\section{Material y Métodos}

Plan Operacional. Hubo brotes de varicela en dos poblados vecinos; Villa Tambores y Piedra Sola. Villa
Facultad de Medicina, Universidad del Uruguay, Montevideo

Departamento de Pediatria (JQR, MMG, CRO)

Ex Asistente de Clínica Pediátrica. Facultad de Medicina (APP, PDOV)

\section{Ministerio de Salud Pública}

del Uruguay

Director Departamental de

Salud (NFJ)

Departamento de

Epidemiología (ICR)

Recibido: 8 de mayo de 2009 Aceptado: 14 de diciembre de 2009

Correspondencia a: Jorge Quian Rivero jorgeq@internet.com.uy 
Tambores es una localidad situada en el límite entre dos departamentos de Uruguay, Tacuarembó y Paysandú, a 440 kilómetros de la capital, Montevideo. Tiene aproximadamente 1.378 habitantes. Se la eligió para la investigación dado que es la localidad que denunció el mayor número de casos.

Cuenta con dos escuelas: la número 64 (escuela A) con 134 alumnos de 6 a 13 años; la número 92 (escuela $\mathrm{B}$ ) con 111 alumnos de 4 a 14 años. Cada una de las escuelas tiene una amplia comunicación entre las aulas de enseñanza y un espacio de esparcimiento en común para todos los alumnos en cada una de ellas.

El pueblo tiene un jardín de infantes, el número 155 al que asisten 70 niños de entre 3 y 5 años.

Además cuenta con un centro de enseñanza secundaria con 250 alumnos.

Definición de brote. Se utilizó la definición entregada por el Ministerio de Salud Pública (MSP) del Uruguay: "aumento inusual del número de casos o la presencia de 2 ó más casos relacionados epidemiológicamente, de aparición súbita y diseminación localizada en un espacio específico".

Diagnóstico de varicela. El diagnóstico de la enfermedad fue hecho en todos los casos por médico, como la presencia de las lesiones características de maculopápulas y vesículas.

Evaluación clínica. Se hizo diagnóstico de varicela en niño vacunado, (en inglés denominado: breakthrough varicella) cuando el paciente desarrolló la enfermedad luego de 42 días de haber recibido la vacuna; en caso contrario, se consideró como varicela en no vacunado ${ }^{9}$.

Cuatro de los integrantes del equipo investigador (MM, $\mathrm{CR}, \mathrm{PD}$ y AP) realizaron consultas telefónicas con las directoras de las escuelas y con muchas de las madres de los casos llenando una ficha con las características de la enfermedad; posteriormente dos de ellas (AP y PD) concurrieron a Villa Tambores donde se registró la edad, el sexo el antecedente de haber o no padecido la enfermedad, y se revisaron manualmente los carné de inmunizaciones de todos los niños escolares y del jardín, constatando si habían recibido la vacuna anti-varicela y, en caso afirmativo, la fecha de administración.

Se tuvo entrevistas con las madres y se interrogó nuevamente sobre el número aproximado de lesiones y la necesidad de medicación u hospitalización.

Se investigaron solamente los casos de los niños escolarizados.

Se clasificó a la varicela como leve ( $<50$ lesiones $)$, moderada (entre 51 y 150) o grave (mas de 150 lesiones o que requiriera hospitalización).

Se consideró fiebre temperatura de $\geq 38^{\circ} \mathrm{C}$ axilar.
Efectividad de la vacuna. En la población de las escuelas y el jardín de infantes, se calculó la tasa de ataque en no vacunados (TANV) como el número de casos de varicela en no vacunados dividido por el número total de niños no vacunados sin historia previa de varicela.

La tasa de ataque en vacunados (TAV) se calculó como el número de casos de varicela en vacunados dividido por el número total de niños vacunados.

La efectividad de la vacuna se calculó según la siguiente fórmula:

\section{$(\underline{\mathrm{TANV}-\mathrm{TAV}}) \times 100 .{ }^{10}$ TANV}

La vacuna utilizada en el Uruguay desde el inicio de la aplicación ha sido Varilrix ${ }^{\circledR}$, de GlaxoSmithKline.

Acciones de control de brote. Se evaluaron las actividades desarrollados por el MSP y la efectividad de las mismas solicitando a las autoridades sanitarias información sobre las acciones emprendidas.

\section{Resultados}

El brote comenzó el 8 de mayo y se extendió hasta el 30 de junio entre las semanas epidemiológicas 15 y 26 .

Se encuestaron 315 niños, 134 de la escuela A, 111 de la escuela B y 70 del jardín de infantes. En la escuela A, una niña de 11 años ingresó a la misma una vez superado el brote, estaba vacunada y no se contabilizó; en otra niña de 9 años, sus familiares habían extraviado el carné de vacunación y no existía constancia en la escuela de las vacunas por lo que también se excluyó del análisis. En total eran entonces, 313 niños.

El primer caso confirmado de varicela fue el de una niña de 9 años, no vacunada, que no había salido del pueblo y que desarrolló una varicela característica diagnosticada por médico.

Un niño de 6 años, previamente vacunado, a fines de abril viajó con sus padres fuera del pueblo y días después desarrolló una erupción cutánea de menos de 20 lesiones, algunas de ellas vesículas, sin fiebre, vistas por médico e interpretadas como alérgicas. Este niño siguió concurriendo a la escuela y era primo conviviente del primer caso confirmado. Se consideró que fue quien introdujo la enfermedad en el pueblo.

En la escuela A, 70 de los niños estaban vacunados, hubo 15 casos de varicela, 3 en vacunados. En un niño de 10 años no fue posible conocer su CEV; se consideró vacunado para los efectos del estudio; 27 tenían historia previa de varicela (ninguno fue caso).

En la escuela B, 70 niños estaban vacunados, hubo 19 casos, 7 de ellos en vacunados; 10 tenían antecedentes de varicela (ninguno fue caso) y 2 no lo recordaban. 


\begin{tabular}{|lll|}
\multicolumn{2}{|c|}{ Tabla 1. Algunas características de los niños con } \\
varicela
\end{tabular}

En un niño que fue caso y que se consideró vacunado se desconocen las características de la enfermedad.

En el jardín de infantes los 70 niños estaban vacunados y hubo 3 casos (Figura 1).

En la escuela A, la TANV fue $11 / 35=0,31$; la TAV fue de $4 / 70=0,05$ y la efectividad de la vacuna $84 \%$.

En la escuela B, la TANV fue $12 / 31=0,39$, la TAV fue $7 / 70=0,1$ y la efectividad $74 \%$.

En el jardín de infantes la TAV fue 3/70 =0,04.

La TANV global 23/66 =0,35; la TAV global 14/210= 0,07 lo que determina una efectividad global de $80 \%$.

Fueron de sexo masculino $64 \%$ de los casos.

Otras particularidades del brote se observan en la Tabla 1.

La efectividad de la vacuna para formas graves fue de 100\%; el caso con más de 150 lesiones se presentó en un no vacunado.

Como dato anecdótico, la relación entre el Poblado de Tambores y el de Piedra Sola, es a través de un ómnibus que transporta fundamentalmente trabajadores; el conductor del ómnibus contrajo la varicela y fue el vector que llevó la varicela a Piedra Sola.

Para bloquear el brote el Ministerio de Salud Pública decidió vacunar a todos los residentes de Villa Tambores, a partir del año de edad y hasta los 35 años, que no hubieran recibido la vacuna, sin historia de haber padecido la enfermedad. Se excluyeron mujeres embarazadas y personas inmunocomprometidas. En esas condiciones, entre el 11 y 17 de julio se vacunó un total de 143 personas. De ellas, 8 tenían menos de 10 años de edad, no estaban vacunados y sus familiares no recordaban que hubieran padecido la enfermedad.

Con esta acción se detuvo el brote.

\section{Discusión}

La vacuna anti-varicela ha demostrado una efectividad global de $80 \%$ y de $100 \%$ para casos graves.

Esta es la primera vez que en Uruguay se documenta un brote de varicela luego de la introducción masiva de

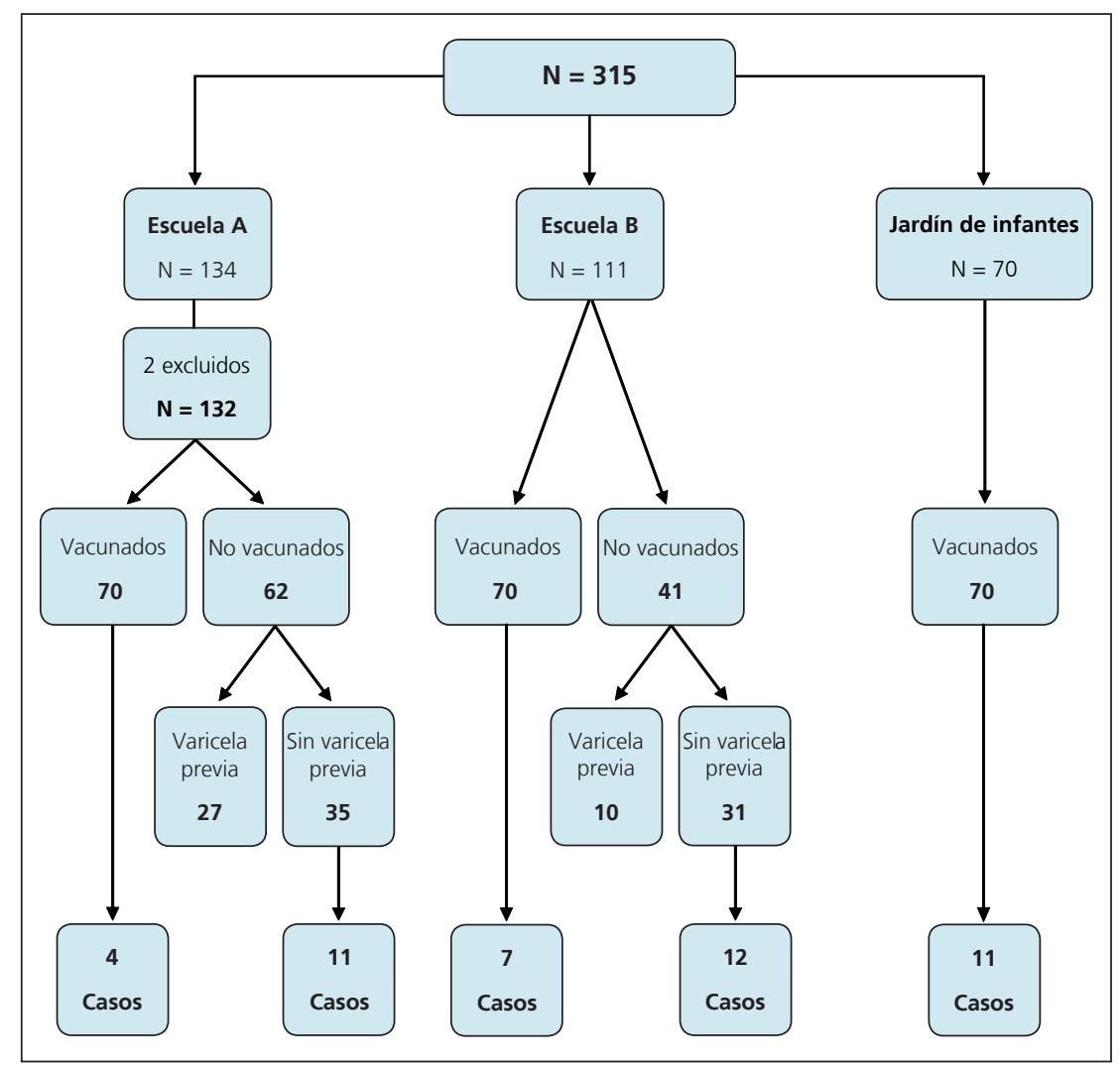

Figura 1. Distribución de los niños analizados según institución e historia inmunitaria.

la vacunación, con determinación de la efectividad de la vacuna y con bloqueo del mismo a través de aplicar la vacuna a quienes no tenían antecedentes de vacunación ni de la enfermedad.

La presencia de un brote en un país con tasas tan altas de inmunización debe promover la investigación de sus posibles causas

Uno de los principales problemas que se plantea actualmente es reconocer la varicela clínicamente en niños vacunados ${ }^{11}$. El primer caso de este brote fue confundido por el médico tratante con alergia. Esto impidió tomar medidas para poder evitar la diseminación de la infección.

Otra de las investigaciones necesarias, es si se cumple con la cadena de frío o si algún lote de la vacuna fue ineficaz.

El encontrar una efectividad como la hallada descarta esas posibilidades.

Diferentes publicaciones han mencionado efectividades que oscilan en $86 \%$ para todas las formas de varicela y $100 \%$ para las formas moderadas y graves $^{12}, 83 \%{ }^{13}$, entre $85 \%$ global y $97 \%$ para formas graves ${ }^{14}$ y por último una revisión de varios artículos ${ }^{15}$ da resultados globales de $84,5 \%$ para todas las formas y $100 \%$ para las graves. Los hallazgos de la presente investigación concuerdan 
con los precedentes.

Quizá halla que investigar si es mejor vacunar a los 15-18 meses en vez de hacerlo a los 12 , habría opiniones de que con este cambio aumenta la seroconversión.

Este brote permitió, a su vez, comprobar el alto porcentaje de niños vacunados; en el jardín de infantes donde concurren niños de entre 3 y 5 años, la totalidad estaba vacunada y solamente 3 de los 70 enfermaron.

Se observó también lo que otros autores describen; hay un desplazamiento de la enfermedad hacia edades mayores ${ }^{16}$. Trece de los que desarrollaron la enfermedad tenían menos de 9 años de edad, de los cuales 9 estaban vacunados; fueron mayores de 9 años 23 niños, de los cuales 19 no estaban vacunados. Esto debería hacer plantear, en países como Uruguay donde no se hizo política de "catch-up" con la vacuna y donde se sigue aplicando una sola dosis, vacunar a adolescentes que no hayan recibido la vacuna y que no tengan historia clínica de varicela. Estos seguramente serán pocos ya que los estudios de prevalencia de anticuerpos en Uruguay, mostraron que a los 10 años, tenían seroconversión $72 \%$ de los estudiados ${ }^{17}$.

En el estudio de los aspectos clínicos, los vacunados tuvieron menos fiebre, menos lesiones que los no vacunados. Tampoco hubo complicaciones en alguno de los dos grupos.

Resultó muy interesante que ninguno de los niños cuyos familiares dijeron que habían tenido la enfermedad, repitió la varicela.

Con respecto a la segunda dosis de la vacuna antivaricela, los autores consideran que debido al costo de la misma, sería conveniente invertir en otras vacunas, ya que se ha comprobado reiteradamente que si bien se puede ver varicela en vacunados, la vacuna protege en $100 \%$ contra las formas graves.

En cuanto a las medidas dispuestas por las autoridades sanitarias para bloquear el brote, fueron adecuadas. Hay experiencia en la región en las mismas ${ }^{18}$.

Agradecimientos. A las directoras de las escuelas, Maestras Lidia Paris y Gladis Vilcher. Al promotor de Salud Wilman Barrales. A Ricardo W Ruttimann por sus valiosos aportes.

\section{Resumen}

Un brote de varicela acaeció en un pueblo uruguayo que había introducido la vacunación anti-varicela en 1999 y tenía altas coberturas de vacunación. Objetivo: Investigar las causas del brote, la efectividad de la vacunación y evaluar las medidas adoptadas. Material y Métodos: Se investigó los casos ocurridos en jardines de infantes y colegios. Se revisó el carné de vacunación de cada niño además de averiguar por historia previa de varicela, las características clínicas de los casos y el resultado de las medidas adoptadas. Se hizo una estimación de la efectividad de la vacuna. Resultados: Se presentaron 37 casos de varicela, 14 de los cuales habían recibido la vacuna, en una población total de 313 niños. La efectividad global de la vacuna fue de $80 \%$, y de $100 \%$ para los casos graves. Se constató un desplazamiento de la enfermedad hacia edades mayores; además, los casos en vacunados tuvieron una tendencia a presentar menos fiebre y un número menor de lesiones. La vacunación de aquellos que no habían tenido la varicela y no estaban vacunados antes, detuvo el brote epidémico. Comentarios: Se demostró la efectividad de la vacuna. La política de vacunación debiera evaluar si es necesario proceder a la vacunación "de rescate" en adolescentes no vacunados y que no exhiben el antecedente de haber padecido la varicela.

\section{Referencias}

1.- Tugwell B, Lee L, Gillette H, Lorber E, Hedberg K, Cieslak P. Chickenpox outbreak in a highly vaccinated school population. Pediatrics 2004; 113: 455-9.

2.- Committee on Infectious Diseases. Recommendations for the use of live attenuated varicella vaccine. Pediatrics 1995; 95 (5): 791-6.

3.- MMWR Morb Mortal Wkly Rep. Varicella -related deaths- United States, 2002, 2003 Jun 13, 52 (23): 545-7.

4.- Quian J, Dall'Orso P, Romero C, Cerisola A, Protasio A. Análisis de los resultados de la inmunización universal a los 12 meses de edad con vacuna de varicela a través de las consultas externas y las hospitalizaciones en el Centro de Referencia Pediátrico de Uruguay. Vacunas 2005; 6: 77-81.

5.- Quian J, Ruttimann R, Romero C, Dall'Orso P, Cerisola A, Breuer T, et al. Impact of universal varicella vaccination in 1-year-olds in Uruguay 1997-2005. Arch Dis Child 2008; 93: 845-50.

6.- Arvin A. Gershon A. Control of varicella. Why is a two-dose schedule necessary? Pediatr Infect Dis J 2006; 25 (6): 2006.

7.- CDC. Prevention of varicella. Recommendations of the Advisory Committee on Immunization Practices (ACIP). MMWR Morb Mortal Wkly Rep 2007; 56 (RR 04): 1-40.

8.- Ministerio de Salud Pública. Dirección General de la Salud. División Epidemiología. Boletín Epidemiológico Septiembre 2008; Vol.2.

9.- Buchholz U, Moolenaar R, Peterson C,
Mascola L. Varicella outbreaks after vaccine licensure: should they make you chicken? Pediatrics 1999; 194: 561-3.

10.- Haddad M, Hill M, Pavia A, Green C, Jumaan A, De A, Rolfs R. Vaccine effectiveness during a varicella outbreak among schoolchildren: Utah, 2002-2003. Pediatrics 2005; 115: 1488-93.

11.- Galil K, Fair E, Mountcastle N, Britz P, Seward J. Younger age at vaccination may increase risk of varicella vaccine failure. $\mathrm{J}$ Infect Dis 2002; 186: 102-5.

12.- Izurieta S, Strebel P, Blake P. Postlicensure effectiveness of varicella vccine during an outbreak in a child care center. JAMA 1997; 278: 1495-9.

13.- Clements D, Moreira S, Coplan P, 
Bland C, Walter E. Postlicensure study of varicella vaccine effectiveness in a day-care setting. Pediatr.Infect. Dis J 1999; 18: 1047-50.

14.- Vázquez M, LaRussa P S, Gershon A A, Steinberg S P, Freudigman K, Shapiro E D. The effectiveness of the varicella vaccine in clinical practice. N Engl J Med 2001; 344 (13): 955-60.
15.- Seward J. Marin M. Vazquez M. Varicella vaccine effectiveness in the US vaccination program: a review. J. Infect. Dis. 2008; 197: S82-S89.

16.- Guris D, Jumaan A. Mascola L. Watson B. Zhang J.Chaves S. et al. Changing varicella epidemiology in active surveillance sites_United Status, 1995-2005. J. Infect .Dis 2008; 197 (Suppl 2): S71-S75.
17.- Quian J, Ruttimann R, Matrai L. Prevalencia de anticuerpos contra varicela en una población de Montevideo, Uruguay. Arch Ped Urug 2004; 75 (2): 133-8.

18.- Gentile A, Marcó del Pont J. Martinez E, Pueta G, Joaquín W, Castrillón S, et al. Efectividad de la vacuna anti-varicela-zoster como profilaxis post-exposición. Arch Argent Pediatr 2002; 100 (1): 25-30. 